\title{
Kernos
}

Revue internationale et pluridisciplinaire de religion grecque antique

18 | 2005

Varia

\section{Il corpo mitico dell'eroe. Eroi e santi nella rappresentazione di un cristiano d'Oriente}

\section{Chiara Cremonesi}

\section{OpenEdition \\ Journals}

\section{Edizione digitale}

URL: http://journals.openedition.org/kernos/1914

DOI: 10.4000/kernos.1914

ISSN: 2034-7871

\section{Editore}

Centre international d'étude de la religion grecque antique

\section{Edizione cartacea}

Data di pubblicazione: 1 gennaio 2005

Paginazione: 407-420

ISSN: 0776-3824

Notizia bibliografica digitale

Chiara Cremonesi, « II corpo mitico dell'eroe. Eroi e santi nella rappresentazione di un cristiano

d'Oriente », Kernos [En ligne], 18 | 2005, mis en ligne le 14 septembre 2011, consulté le 06 mai 2019.

URL : http://journals.openedition.org/kernos/1914 ; DOI : 10.4000/kernos.1914 


\title{
Il corpo mitico dell'eroe. Eroi e santi nella rappresentazione di un cristiano d'Oriente
}

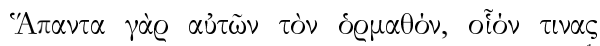

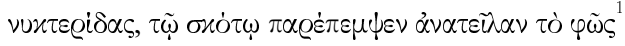

\begin{abstract}
Résumé : Le corps mythique du héros. Les dieux et les héros grecs sont refoulés dans les ténèbres comme des chauve-souris, Jésus est la lumière victorieuse : ce sont les mots de Théodoret dans sa Graecarum affectionum curatio, l'un des plus précieux témoignages de l'apologétique chrétienne. Dans cette œuvre, l'évêque de Cyr parle aux païens de leurs héros et en particulier de l'iniquité du culte hérö̈que : cette représentation ne s'explique pas seulement par les topoi de la rhétorique chrétienne, mais aussi par rapport à la réflexion de Théodoret sur l'endurance des ascètes. Ce sont en effet les corps qui " posent le plan de l'énonciation » : les corps faibles des héros, destinés à être vaincus par la souffrance, incapables de supporter la douleur et la maladie, face aux corps trempés par la karteria des martyrs et des ascètes, qui prennent sur eux la souffrance et en font ainsi la «marque » de la sainteté, en actualisant par leur hyponomè celle du Christ. La représentation que Théodoret donne des corps vaincus des héros «marque » l'éloignement de la vera religio du mythe; l'auteur esquisse des modalités nouvelles de la relation entre l'humain et le divin, et il décrit un scénario, où les corps sont aussi éloquents que le logos.
\end{abstract}

Abstract: The mythical body of the hero. The Greek heroes and gods are vanished into darkness, like bats; actually, Jesus Christ is the victorious light: these are Theodoret's words in the Graecarum affectionum curatio, one of the most important texts of Christian apologetics. In his work, Theodoret offers to pagans a picture of their own heroes, in order to demonstrate the iniquity of the heroic cult. Evidences of such a statement is provided through Christian rhetorical topoi. However, an important argument against the heroic cult is the practice of ascetic endurance. According to Theodoret, on the one hand, the bodies of pagan heroes, who are unable to endure suffering and diseases, are doomed to be defeated by pain. On the other hand, martyrs and ascetics are able to endure suffering and to assume it, just through their bodies, hardened by karteria, so that they turn it into the mark of holiness. In this sense, Christ's hypomone is re-enacted by the ascetic hypomone. Therefore, the heroic bodies, defeated by their own inability to endure suffering, stress the distance between vera religio and myth. So, the Christian author displays a new kind of relationship between humanity and divinity, describing a scene in which bodies are as meaningful and eloquent as logos.

Con queste parole e l'augurio che i suoi lettori possano partecipare della luce, Teodoreto conclude la Graecarum Affectionum Curatio, una delle

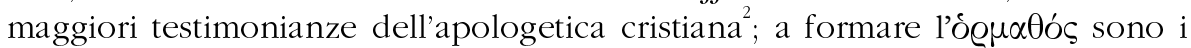
demoni pagani, pipistrelli ricacciati nelle tenebre: su tutti si staglia Asclepio, il solo cui sia concesso nome nel momento della celebrazione della vittoria,

\footnotetext{
1 Theodoretus, Graecarum affectionum curatio XII, 97.

2 Si tratta di una delle prime opere di Teodoreto, probabilmente redatta prima del Concilio di Efeso (431): $c f$. a tale proposito P. CANIVET (a cura di), Théodoret de Cyr. Thérapeutique des maladies helléniques I, Paris, Cerf, 1958 (Sources Chrétiennes), p. 28-31.
} 
definitiva, del Salvatore. Se infatti Teodoreto rifiuta al medico della mitologia greca lo statuto di divinità soterica ${ }^{3}$, recuperandone, anche grazie alla selezione accorta delle fonti pagane, la dimensione eroica, solo ad Asclepio egli sembra del resto riservare il ruolo di antagonista del Cristo, per quanto esso sia, di necessità, incompiuto, abdicato nel momento stesso della croce e della passione del figlio di Dio.

Se la scelta di Teodoreto di concedere ad Asclepio la guida della tenebrosa schiera dei demoni pagani può sembrare ovvia per ragioni retoriche da un lato, per ragioni di «concorrenza » soterico-cultuale dall'altro ${ }^{4}$, la conclusione della Graecarum Affectionum Curatio assume un valore peculiare in rapporto alle modalità della rappresentazione cristiana del corpo mitico "pagano », laddove essa diviene peraltro testimonianza della mutata relazione tra umano e divino intervenuta con il Cristianesimo.

Non ci interessa qui ripercorrere la misura del rapporto tra il paradigma eroico pagano e il modello della santità cristiana nella rappresentazione retorica tardo-antica ${ }^{5}$ ma piuttosto uno dei modi in cui Teodoreto «segna la distanza » tra cristianesimo e paganesimo: egli sembra farlo anche attraverso la contrapposizione tra «modelli» corporei. Da un lato infatti l'autore antiocheno si premura di definire la differenza tra i corpi dei cristiani e quelli dei pagani; dall'altro insiste sulla distanza tra i corpi degli eroi pagani da una parte e quelli degli apostoli e dei santi cristiani dall'altra: i due livelli del confronto si presentano strettamente correlati tra loro, laddove Teodoreto non individua infatti solo differenti «morfologie » somatiche ma diversi e opposti modi di gestire il corpo.

L'attenzione del vescovo di Cirro nei confronti dei «corpi » eroici si inserisce del resto all'interno di uno scenario, qual è quello della letteratura tardoantica pagana e cristiana, in cui il lessico relativo al corpo, alle malattie e alla cura ricorre estremamente di frequente, rivestendo sì valenze metaforiche già presenti nella letteratura classica da un lato, in quella vetero e neo-

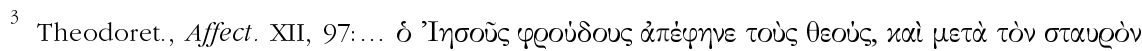

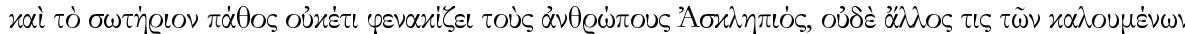

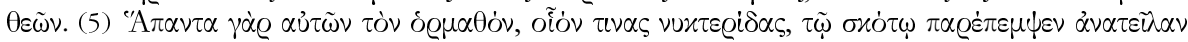

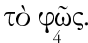

Cf. a tale proposito E.J. EDELSTEIn, L. EDELSTEIn, Asclepius. A collection and interpretation of the testimonies II, Baltimore, The Johns Hopkins Press, 1945, p. 133: "It seems that the Christians themselves realized that in Asclepius they faced their strongest enemy, the most dangerous antagonist of their Master. »

Rinvio qui soltanto a G. Freyburger, L. Pernot (a cura di), Du héros païen au saint chrétien. Actes du colloque organisé par le Centre d'Analyse des Rhétoriques Religieuses de l'Antiquité (C.A.R.R.A.). Strasbourg, $1^{\text {er }}-2$ décembre 1995, Paris, Institut d'Études Augustiniennes, 1997 (Collection des Études Augustiniennes), e in particolare a G. FreYburger, L. PERnot, « Avantpropos », p. 6: «Dans quelle mesure le christianisme a-t-il inventé de nouveaux modèles, dans quelle mesure a-t-il recyclé, converti les modèles préexistant ? Les éléments de permanence et de fracture conduisent à une méditation sur les archétypes de la rhétorique religieuse. Le mots de passage, de continuité, de transition ou de rupture entre excellence païenne et excellence chrétienne ne décrivent qu'une face de la réalité. Il faut parler aussi de chevauchements et de mélanges. » 
testamentaria dall'altro -, ma rivelandosi soprattutto testimone di un mondo in cui il corpo si pone al centro della comunicazione simbolica; in cui la malattia non è mai ridotta a puro evento nosologico, non sussistendo nella tradizione iatrica antica quell'implicito presupposto teorico dell'inutilità e del «nonsenso » della malattia, che caratterizza il razionalismo medico-scientifico contemporaneo.

Se già con la techne ippocratica ebbe origine il metodo sperimentale e diagnostico, la ricerca di una causa naturale delle patologie, e l'individuazione di cure rispondenti anch'esse a leggi di natura, ciò non vuol dire che non vi fosse implicito un «sens du mal »: lo stato dell'individuo era strettamente correlato alla struttura e alle condizioni della realtà cosmica di cui egli faceva parte e pertanto la rappresentazione dell'evento nosologico, così come della salute, si caricava di forti implicazioni simboliche.

Si tratta di una generalizzazione che, come tale, non rende ragione anche delle profonde divergenze tra i sistemi medico-epistemologici dell'antichità , i quali sembrano però condividere un comune criterio discriminante tra salute $\mathrm{e}$ malattia, ovvero il principio della "giusta mescolanza», un a priori, evidentemente non sperimentale, che trova una corrispondenza nell'ideale peculiarmente greco della « esatta proporzione ${ }^{8}$. "

Ne è testimone lo stesso Galeno, che nel II sec. d. C. affermava: «Quasi tutti i miei predecessori hanno definito la salute come la buona mescolanza (eucrasia) e come la proporzione (simmetria) degli elementi ». Egli stesso, grande sistematizzatore e al tempo stesso innovatore del pensiero medicoscientifico, aderiva a questo principio nel momento in cui concepiva la salute come equilibrio, o meglio come una serie di equilibri successivi, connessi gli uni agli altri.

Cf. M. Augé, C. Hertzlich (a cura di), Le sens du mal. Anthropologie, histoire, sociologie de la maladie, Paris, Éditions des Archives Contemporaines, 1984.

7 Sicuramente non rende ragione degli «anti-epistemologi», ovvero gli «empirici », setta medica ellenistica che, sviluppatasi ad Alessandria ed influenzata dalla filosofia scettica, rifiutò appunto alla medicina lo statuto di scienza, ritenendola una terapeutica totalmente aliena alla speculazione. $C f$. a proposito della nozione di "setta medica ", nata ad Alessandria nel III sec. a. C., D. Gourevitch, "Le vie della conoscenza: la medicina nel mondo romano", in M.D. GRMEK (a cura di), Storia del pensiero medico occidentale I. Antichità e Medioevo, Roma/Bari, Laterza, 1993, p. 121 ss. Gli stessi trattati del Corpus Hippocraticum sono del resto stati distinti in rapporto all'orientamento intellettuale dei diversi autori, medici teorici, medici empirici e medici che tentavano di conciliare teoria ed osservazione: $c f$. a tale proposito L. BOURGEY, Observation et expérience chez les médecins de la Collection Hippocratique, Paris, Vrin, 1953, p. 47-78.

Cf. a tale proposito S. NATOLI, L'esperienza del dolore. Le forme del patire nella cultura occidentale, Milano, Feltrinelli, 2001' ${ }^{2}$, in particolare p. 124, dove l'autore afferma: «La giusta mescolanza rimaneva, comunque, il criterio discriminante tra salute e malattia, indipendentemente dai mezzi che si ritenevano adatti a restaurarla qualora essa venisse meno. L'equilibrio rappresenta una funzione dinamica che integra insieme microcosmo e macrocosmo, determinazione singola e contesto, organismo e ambiente... Ciò che vale per il cosmo, vale anche per l'uomo, che a tale normativa è vincolato per il fatto stesso d'essere un caso particolare della physis, definito secondo un suo status ed un suo equilibrio. La determinazione dell'equilibrio compete, quindi, anche all'individuo che soffre, sia come entità fisica che come soggetto morale. » 
Il 'dogma' della 'giusta mescolanza', che si conserva nella medicina bizantina di derivazione galenica, mette a nudo una scienza iatrica in cui empirismo e determinismo non sono necessariamente alternativi ad una volontà di 'significare' il cosmo, e in cui la malattia, di conseguenza, non è ridotta a puro evento nosologico, ma diviene al contrario 'significante'.

Se l'equilibrio infatti rappresenta una funzione dinamica, che integra microcosmo e macrocosmo, esso si configura, nello stesso tempo, come realtà condenda: deve essere cioè mantenuto, o ripristinato, anche attraverso norme di retto comportamento, che trovano quindi fondamento nello stesso sistema epistemologico. A ciò è connessa quella funzione profilattica che sin dall'inizio la medicina greca ha assunto e che diviene particolarmente chiara nell'episteme iatrica di tradizione galenica della Siria bizantina': se il male non doveva semplicemente essere rimosso attraverso la cura, ma prevenuto, tale azione preventiva si traduceva di fatto in un orientamento del bios individuale e collettivo, non scevro da componenti di natura, a seconda dei differenti contesti storico-culturali, rituali ed etiche, tanto che il lessico della medicina si fa vicino, o meglio si intreccia con quello del nomos. Ciò accadeva a maggior ragione in quella particolare relazione terapeutica che intercorreva tra gli asceti cristiani taumaturghi e i fedeli che si raccoglievano intorno a loro nella speranza di ottenere una guarigione: tale relazione viene descritta magistralmente dallo stesso Teodoreto nell'Historia Religiosa ${ }^{10}$, opera in cui vengono celebrate le gesta degli asceti di Siria. In tale contesto malattia e attività terapeutica sembrano connotarsi più che mai come «fattori sociali», nei termini in cui la relazione medico-paziente/asceta-fedele interviene sul bios degli individui, fondando per essi una nuova - o una rinnovata - adesione al messaggio evangelico ed assumendo quindi un'ampia ricaduta: non solo essa mette in gioco legami parentali e sociali, ma, concepita nei termini di azione apostolica, agisce sul « corpo della Chiesa», progressivamente rafforzandolo o accrescendolo attraverso la conversione degli infedeli.

Proprio polemizzando contro il politeismo greco Teodoreto si sofferma sulla questione dei corpi sani e perfetti, laddove si trova ad affermare la superiorità del regime monarchico rispetto all'anarchico e al poliarchico, supremazia perfettamente speculare a quella del monoteismo sul politeismo.

Teodoreto esordisce infatti ricordando da una parte l'ammirazione comune nei confronti dei corpi ben costituiti - $\alpha \varrho \tau \iota \mu \varepsilon \lambda \tilde{\eta}-$ ed integri - $\alpha \ddot{n} \eta \varrho \alpha-$, vale a dire quei corpi che preservano intatto e perfetto l'armonioso insieme ricevuto dalla natura; ribadendo dall'altra la comune ripulsa nei confronti dei corpi che « hanno qualcosa in più » o «qualcosa in meno »; questi ultimi - afferma il

\footnotetext{
215.

9 Cf. G. STROHMAiER, «La medicina nel mondo bizantino e arabo », in GRMEK, o.c., p. 167-

L'opera viene datata alla prima metà del quarto decennio del V secolo: $c f$. a tale proposito A.J. Festugière, Antioche païnne et chrétienne, Paris, de Boccard, 1959 (BEFAR), p. 245 P. CANIVET, Le monachisme syrien selon Théodoret de Cyr, Paris, Éditions Beauchesne, 1977 (Théologie Historique), p. 32; H. LEPPIN, «Zum kirchenpolitischen Kontext von Theodorets Mönchgeschichte », Klio 78 (1996), p. 212-230.
} 
vescovo di Cirro - vengono comunemente definiti $\tau \dot{\varrho} \varrho \alpha \tau \alpha$, mostri ${ }^{11}$ : appartenendo alla dimensione dell'eccezionalità, che esorbita dall'equilibrio, essi si iscrivono nell'ambito dell'anomalia mostruosa.

Se v'è per Teodoreto correlazione simbolica tra modelli somatici e tipologia di governo - l'anarchia rinvia ai corpi privi di qualcosa, la poliarchia ai corpi che hanno qualcosa in più, la monarchia ai corpi sani -, parallela corrispondenza si viene ad instaurare tra sistemi di governo e sistemi religiosi: se i corpi sani sono di necessità quelli cristiani, quelli malati appartengono agli empi e ai miscredenti, in primis ai pagani, sia quelli che non hanno conosciuto la fede salutare e salvifica, sia quelli che l'hanno rifiutata.

Il vescovo di Cirro invita quindi i suoi lettori a seguire tale regola: denominare come sani e perfetti coloro che hanno abbracciato la vera teologia -

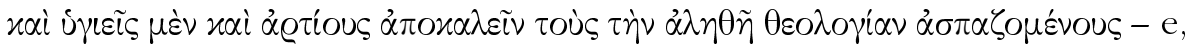
al contrario, qualificare come mutilati - $\alpha \nu \alpha \pi \hat{n} \varrho O u \varsigma \delta^{\prime} \alpha \tilde{3} \pi \varrho O \sigma \alpha \gamma O \varrho \varepsilon u ́ \varepsilon l \nu$ - non solo coloro che non credono in alcun dio, ma anche coloro che hanno suddiviso la maestà divina e che hanno posto sullo stesso piano il Demiurgo e la creazione ${ }^{12}$.

E qui entrano finalmente in scena gli eroi: se i corpi dei pagani sono per Teodoreto insani e imperfetti, «insalubri» sono infatti anche i demoni che essi adorano; questi ultimi infatti eccedono dalla metriotes e si allontanano da quel buon governo del corpo necessario al mantenimento e alla conservazione della salute; se i corpi delle divinità sono volgarmente schiavi delle passioni umane, lo sono a maggior ragione quelli degli eroi.

Teodoreto si sofferma in particolare su quella che sembra essere per lui una vera e propria «coppia logica» costituita da Eracle ed Asclepio, cui l'autore cristiano riconosce il medesimo statuto, appunto quello di « eroe », di uomo divinizzato.

Il vescovo di Cirro li ricorda dopo aver elencato le differenti creature che i pagani adorano in luogo del Creatore, nonostante quest'ultimo le abbia forgiate volutamente «difettose », caduche, incompiute e imperfette, proprio perché gli uomini non incorressero nell'errore di adorare l'oggetto sbagliato: il sole o la luna, che, nonostante garantiscano quotidianamente benefici agli uomini, possono essere oscurati dalle nuvole o eclissarsi nel mezzo del giorno o della notte; l'aria, che, se è indispensabile per vivere, al tempo stesso trasmette malattie pestilenziali, non permanendo immutabile ma essendo

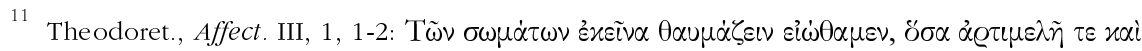

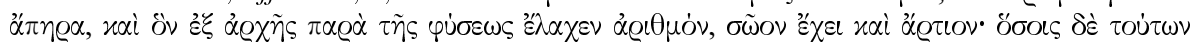

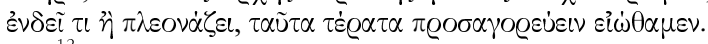

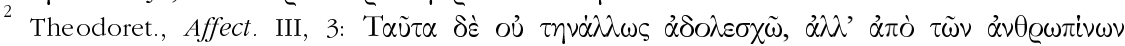

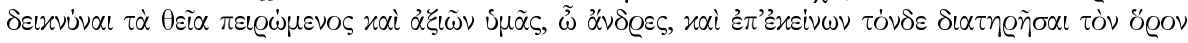

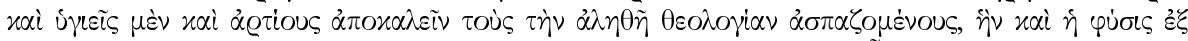

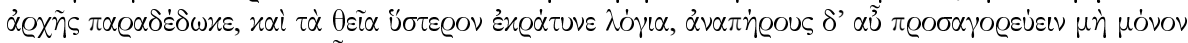

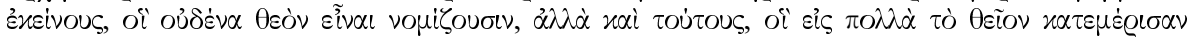

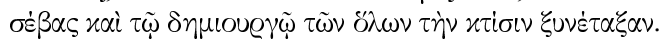


soggetta a variazioni ed alterazioni; la terra, che fornisce sì i frutti ma patisce a volte la sterilità, emettendo irrevocabili sentenze di morte.

Se gli elementi naturali - afferma il vescovo di Cirro - furono i primi ad essere venerati come dei da Fenici, Egiziani e Greci, in un secondo momento furono divinizzati coloro che avevano compiuto delle belle azioni, che si erano comportati coraggiosamente in guerra, che avevano apportato qualche innovazione in agricoltura o trovato una cura per le malattie del corpo.

All'interno di questa schiera Teodoreto colloca Eracle ed Asclepio; il primo - sostiene il vescovo di Cirro - fu divinizzato dai Greci per il suo coraggio e la sua forza:

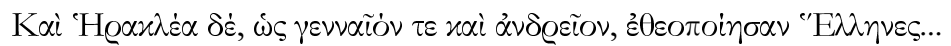

il secondo perché scopritore della scienza medica:

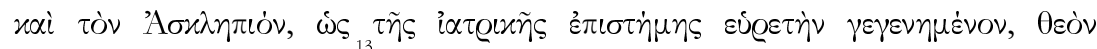
$\tau \varepsilon \lambda \varepsilon u \tau \dot{\eta} \sigma \alpha \nu \tau \alpha \pi \varrho O \sigma \eta \gamma \dot{\varrho} \varrho \varepsilon \cup \sigma \alpha \nu{ }^{13}$.

Eracle sembra in qualche modo rientrare nella schiera dell'imperfezione per eccesso: il suo corpo è quasi mostruoso nell'essere votato al modello della dismisura, pleonasticamente mancante ad infrangere ogni prescrizione rivolta agli uomini, dalla buona misura nella condotta sessuale a quella nella dieta, modello per eccellenza di quella condizione atletica che, secondo Galeno, non esisteva per natura ${ }^{14}$.

Eracle non fu soltanto un uomo assurdamente divinizzato dai Greci, ma fu pessimo tra gli uomini: egli non aveva amato né la temperanza, né la filosofia, ma aveva passato la vita nella dissoluzione e nell'errore ${ }^{15}$.

Scegliendo sapientemente tra le fonti, l'autore cristiano presenta infatti un Eracle uomo, figlio di Alcmena e Anfitrione, sorprendentemente annoverato fra gli dei dai pagani ${ }^{16}$, che a lui dedicarono templi e altari, offrirono sacrifici, intitolarono giorni di festa. Teodoreto descrive il progressivo propagarsi del culto di Eracle come si trattasse di una pestilenza, la malattia dell'errore, che non contaminò soltanto Ateniesi e Spartani, ma tutta la Grecia e la maggior parte d'Europa: il vescovo e intellettuale cristiano presenta la progressiva

13 Theodoret., Affect. III, 26, 3

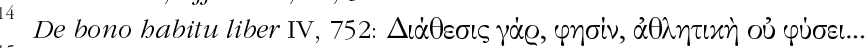

15 Theodoret., Affect. VIII, 15. Teodoreto rifiuta Eracle come esempio di saggezza e giustizia, ruolo attribuitogli da molta della riflessione filosofica sin dal V secolo: $c f$. in generale G.K. GALINSKY, The Herakles Theme. The Adaptations of the Hero in Literature from Homer to the Twentieth Century, Oxford, Blackwell, 1972; cf. anche D.E. AunE, "Herakles and Christ. Heracles Imagery in the Christology of Early Christianity", in D.L. BALCH, E. FERGUSON, W.A. MEEKs (a cura di), Greeks, Romans, and Christians. Essays in Honor of Abraham J. Malherbe, Minneapolis, Fortress Press, 1990, p. 3-19: l'autore si sofferma peraltro sulla questione del rapporto tra l'Eracle pagano e il Cristo dei Vangeli, già affrontata da M. Simon: M. SimON, Hercule et le Christianisme, Paris, Editions Orfphrys, 1955; A. RODIGHIERO, "Morire per amore, indossare la morte », in A. Rodighiero (a cura di), Sofocle. La morte di Eracle (Trachinie), Venezia, Marsilio, 2004, p. 9-31.

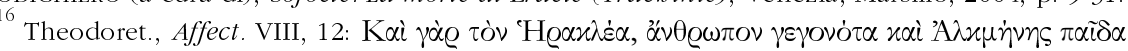

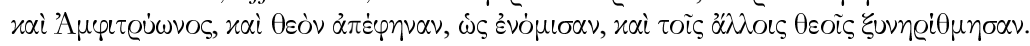


diffusione del culto specularmente al modo in cui descrive in più luoghi delle sue opere il progredire della Buona Novella, che raggiunge i popoli grazie all'azione apostolica ${ }^{17}$.

Se il tema della smoderatezza di Eracle non è evidentemente nuovo, più interessante è il fatto che Teodoreto, quando si trova a descriverne la vicenda, non insista tanto sugli eccessi dell'eroe in vita, quanto piuttosto sulle modalità della sua morte ${ }^{18}$.

L'intellettuale cristiano insiste in particolare sulla causa ultima del decesso di Eracle, vale a dire l'incontinenza: è in conseguenza infatti della propria $\alpha$ x $\propto \alpha \sigma i \alpha$ che il traditore di Deianira ${ }^{19}$ viene involontariamente sottoposto dalla compagna al tormento del chitone avvelenato. Teodoreto insiste sull'incapacità dell'eroe greco di resistere alla sofferenza: è il linguaggio del corpo, del dolore e della malattia ad essere privilegiato. Il vescovo di Cirro ridicolizza la figura di Eracle che, colpito dalla gravissima malattia provocata dal farmaco di

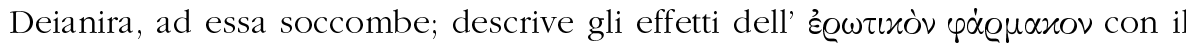
quale la donna unge il chitone, che riscalda talmente il corpo dell'eroe da farlo precipitare in una malattia tremenda. Non essendo in grado di sopportare il dolore - ricorda il vescovo di Cirro - l'eroe si dà la morte nel fuoco:

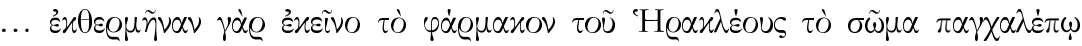

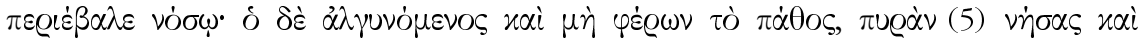

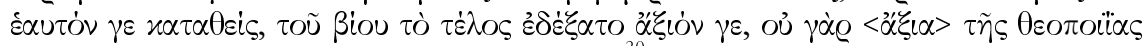

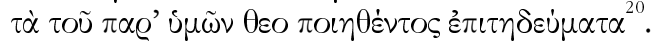

Come possono quindi i pagani - si chiede Teodoreto - attribuire il titolo di salvatore e di protettore ${ }^{21}$, ad un uomo incapace di sopportare la sofferenza, il titolo di « $\ddot{\alpha} \nu \alpha \xi$ » ad un uomo incapace di resistere al dolore ${ }^{22}$ ?

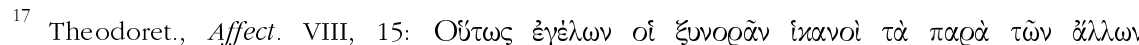

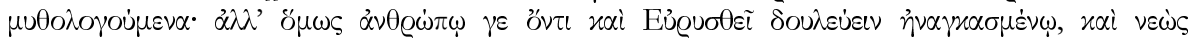

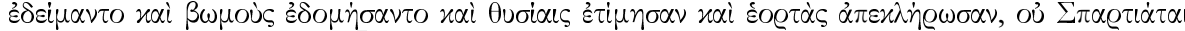

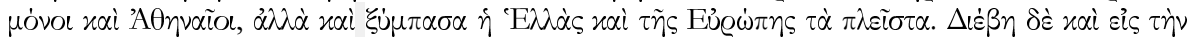

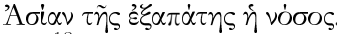

${ }^{18}$ Sulla morte eroica, $c f$. A. BRelich, Gli eroi greci. Un problema storico-religioso, Roma, Ateneo, 1958, p. 80 ss.; $c f$. in particolare p. 87: «A prescindere... da qualsiasi discordanza che può delinearsi apparentemente tra il mito e il culto di un singolo eroe, non si potrà disconoscere la massiccia validità del fatto che se uno dei tratti salienti del culto eroico è la sua frequente localizzazione presso una tomba (e un altro è il rito sacrificale di tipo funebre), uno dei tratti salienti della mitologia eroica è che gli eroi muoiono... »; cf. anche p. 88: «E come nel culto eroico non basta la tomba e non basta il sacrificio di tipo funebre, ma lo stato di morte dell'eroe viene sottolineato spesso da particolari riti di lutto, così neanche il mito si accontenta di riferire che l'eroe è morto; la morte dell'eroe ha quasi sempre un rilievo particolare. »

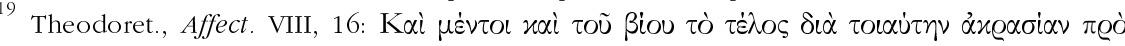

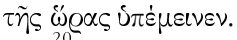

${ }^{20}$ Theodoret., Affect. VIII, 15

${ }^{21}$ Teodoreto riprende il tradizionale epiteto di Eracle $\dot{\alpha} \lambda \varepsilon \xi i x \alpha x \circ$ s, " che tiene lontano i mali ».

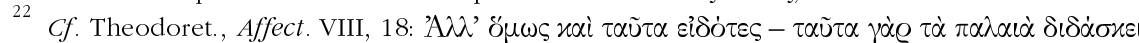

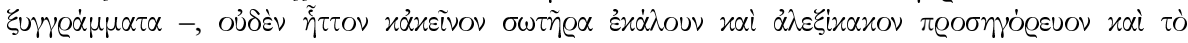

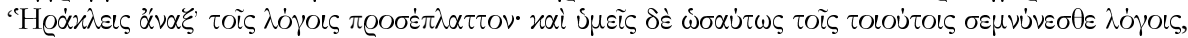

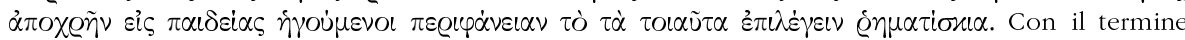


Salvatore può essere soltanto chi, come il Cristo, ha assunto la sofferenza propria e degli uomini; garante di intercessione salvifica solo chi imita il figlio di Dio: la figura di Eracle non si va infatti a contrapporre solo a quella dei martiri, che, al contrario dell'eroe tragicomico di Teodoreto, sopportano con coraggio le prove estreme della tortura e della violenza ultima del supplizio, ma di quegli stessi asceti celebrati dal vescovo di Cirro nell'Historia Religiosa, i quali non solo sopportano con karteria le ferite e i mali più tremendi, ma per i quali la malattia stessa diviene «segno» dell'essere stati prescelti dalla grazia divina e conditio sine qua non per poter divenire strumento di essa.

Se nell'VIII capitolo della Graecarum Affectionum Curatio Teodoreto insiste sulla débâcle di Eracle di fronte al male, nell'opera in cui celebra gli «astri d'Oriente» egli parallelamente si dilunga nella descrizione delle affezioni che tormentano gli asceti: mai ridotte a puro evento nosologico, esse divengono evidenza estrema e inconfutabile della natura umana e, appunto, non demonica del santo.

Non solo: la malattia - vóбos o $\pi \dot{\alpha} \theta$ os che sia -, nel momento in cui viene sconfitta dall'intervento divino, sembra divenire garanzia della stessa capacità taumaturgica dell'asceta e della derivazione di quest'ultima da Dio; la grazia divina, avendo garantito la salute all'asceta, risana, attraverso il guaritore guarito, i malati che a lui si rivolgono, rovesciando in qualche modo il motivo della mitologia eroica dell'ammalato guaritore o del guaritore ammalato ${ }^{23}$, in base al quale chi è guaritore di molte ferite, viene in ultimo ferito inguaribilmente.

Se Teodoreto rappresenta Eracle che soccombe alla propria malattia cercando la morte e che non può quindi in nessun modo configurarsi come terapeuta, al contrario quando si trova a parlare degli «astri d'Oriente » rappresenta la malattia dell'asceta e il superamento di essa come il presupposto, la conditio sine qua non dell'attività terapeutica: ciò è evidente nella stessa scansione narrativa che in Teodoreto sembra, più che rivelare la struttura ideologica, costruirla.

Un esempio chiarificatore in questo senso può essere quello di Giuliano, asceta dell'Osroene che, arrivato ad Antiochia e preceduto dalla sua fama ${ }^{24}$, viene raggiunto da una folla accorsa da ogni parte per soddisfare il desiderio di vedere l'« uomo di Dio » e di ottenere guarigioni. L'asceta rifiuta alla folla la

$\dot{\alpha} \lambda \varepsilon \xi \xi i x \varkappa o \varsigma$ del resto Teodoreto definisce, proprio nelle ultime righe dell'opera il pharmakon da

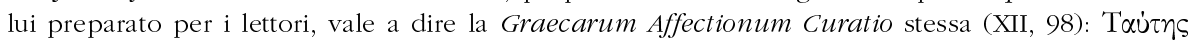

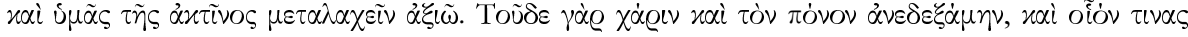

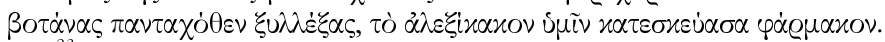

${ }^{23}$ Cf. BRELICH, o.c., p. 118: "Cheiron, guaritore di tante ferite, finisce per essere ferito inguaribilmente (Apollod., II, 85); nell'Iliade (XI, 834 sg.) Podaleirios a un certo momento diventa «bisognoso, anche lui, d'un ottimo medico »... Più noto e chiaro è un altro motivo, diventato proverbiale: "guarisce solo chi ha ferito ». Il più conosciuto dei miti relativi è indubbiamente quello di Telephos che, ferito da Achilleus, non può esser guarito che da Achilleus stesso o dalla sua lancia...»

24 Theodoret., Historia Religiosa II, 18, 2-4 
propria azione terapeutica ${ }^{25}$ e si ritira nelle grotte ai piedi del monte, dove si diceva si fosse ritirato il divino apostolo il beato Paolo ${ }^{26}$ : « ma subito - afferma Teodoreto -, perché tutti sapessero che era un uomo, Giuliano cade preda di un accesso violentissimo di febbre ${ }^{27}$.

L'evento nosologico «significa » la natura umana di Giuliano, ed è direttamente posto in relazione con l'attitudine iatrica dell'asceta: quando quest'ultimo cade preda della febbre, il suo compagno Acacio se ne dispiace, preoccupato per la reazione della folla. Racconta infatti Teodoreto che costui, vedendo la moltitudine riunita, si perse d'animo per il sopraggiungere dell'infermità - $\dot{\alpha} \varrho \omega \sigma \tau i \alpha$ : riteneva infatti che quanti si erano riuniti sarebbero rimasti turbati se avessero saputo della malattia - vóon $\mu \alpha$ - di Giuliano mentre attendevano di ricevere proprio da lui la guarigione $-\theta \varepsilon \varrho \alpha \pi \varepsilon i \alpha$.

Come poteva in effetti la folla, accorsa per ricevere la salute, reagire alla malattia del guaritore? La risposta di Giuliano alle preoccupazioni di Acacio è altrettanto eloquente:

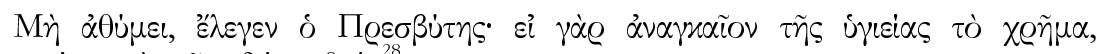

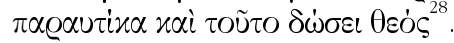

Se la salute era necessaria, Dio gliela avrebbe concessa.

Dopo aver detto questo ad Acacio, Giuliano prega Dio, supplicandolo di donargli la guarigione, qualora essa sia di vantaggio per la folla: la sua preghiera non è ancora terminata che un grande sudore improvviso estingue la fiamma della febbre. Da quel momento Giuliano dà inizio alla sua attività terapeutica, liberando molti da malattie di ogni genere ${ }^{29}$.

Si potrebbe sintetizzare la sequenza narrativa in cinque momenti - in questo come in molti altri casi dell'Historia Religiosa: 1) richiesta-rifiuto di guarigione; 2) malattia dell'asceta; 3) preghiera a Dio; 4) guarigione donata da Dio; 5) inizio dell'attività terapeutica.

L'azione terapeutica di Giuliano è successiva, in qualche modo conse-

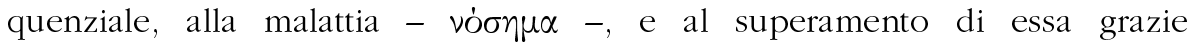
all'intervento divino: perché ammalato, Giuliano è stato salvato e investito dalla grazia divina, e può quindi fungere da tramite di salvezza, fisica e spirituale, tra Dio e gli uomini.

L'asceta dispensa le sorgenti della grazia che dimora in lui - á $\varphi \theta \dot{v} v \omega s_{-}-$, con generosita ${ }^{30}$ : egli condivide il dono divino, quella grazia appunto che, avendogli concesso la salute, gli permette d'essere strumento di guarigione per gli altri.

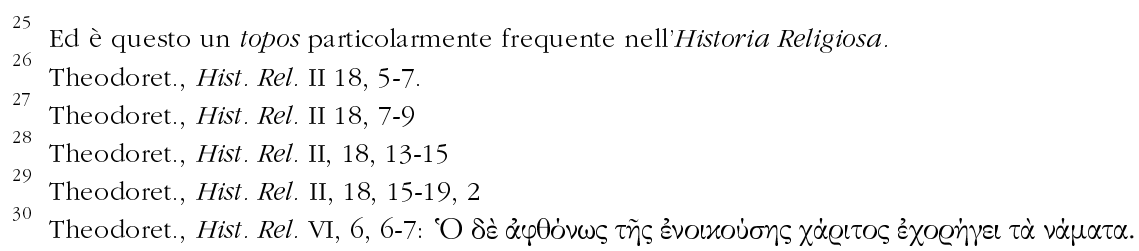


Se Zeus - nella tradizione mitica riportata da Teodoreto - ingenerosamente punisce il «prometeico » Asclepio per aver concesso agli uomini non solo la medicina ma pure la resurrezione, il Dio d'amore guarisce Giuliano e grazie a lui gli uomini; a differenza di Eracle, che, infiammato dal farmaco di Deianira, si butta nel fuoco, l'asceta grazie alla febbre e al sudore "divino », che la estingue, può operare guarigioni, al riparo da ogni sospetto.

Se l'Historia Religiosa racconta quindi di uomini che, pur guarendo, rifiutano d'essere venerati, affermando di essere semplici strumenti divini e ricevendo la stessa capacità taumaturgica dalla grazia divina, la Graecarum Affectionum Curatio ridicolizza uomini cui viene tributato culto nonostante non abbiano, come Eracle, saputo guarire se stessi, o, abbiano, come Asclepio, dovuto apprendere la techne iatrica da Chirone ${ }^{31}$.

Teodoreto insiste del resto più ampiamente sulla «vicenda biografica » di Asclepio rispetto a quanto faccia con Eracle, di cui, come abbiamo visto, racconta piuttosto la morte: richiamandosi ad Apollodoro ma distanziandosi in varie occasioni dall'autore della Bibliotheca ${ }^{32}$, egli recupera la dimensione eroica di Asclepio, trascurando le tradizioni che vogliono di questo ñows

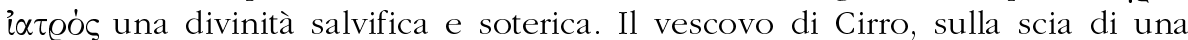
polemica che si trascinava da secoli nell'apologetica cristiana, insiste quindi sulla natura mortale di Asclepio, soffermandosi divertito sul fatto che quest'ultimo avesse avuto bisogno per sopravvivere di una cagna e della pietà dei cacciatori.

Allo stesso modo l'autore cristiano ironizza sul fatto che l'eroe greco non avesse ricevuto il potere di guarire da saggezza o scienza divine, ma da Chirone, il quale gli aveva insegnato una techne, che, in quanto tale, era, in fondo, trasmissibile a chiunque.

Come possono i pagani - si chiede quindi Teodoreto - venerare Asclepio, che non solo imparò da altri, il centauro Chirone, l'arte medica ${ }^{33}$, ma che ai

\footnotetext{
31 Theodoret., Affect. VIII, 20. Teodoreto infatti insiste su quelle fonti pagane che rappresentano un Asclepio che non è sorgente autonoma dell'arte medica avendola egli appresa da Chirone; cf. Brelich, o.c., p. 117: la «linea, infatti, parte da Cheiron, come nel caso di altri arti eroiche..., di modo che la discendenza di Asklepios ne è solo una grossa diramazione: tanto è vero che nell'Iliade che pur conosce tra le fila dei Greci i due figli di Asklepios che vi hanno funzioni di medico, in un caso a Patroklos viene richiesto di guarire Eurypylos di una ferita (11, 828 sgg.) perché egli ha imparato l'arte iatrica da Achilleus che a sua volta l'aveva appresa da Cheiron. Achilleus stesso esercita la propria scienza medica, bendando la ferita di Patroklos, - e con ciò l'intervento guaritore dell'eroe presso un compagno ferito entra come tema obbligato nell'epica. »

Cf. J.C. CARrière, B. Massonie, La Bibliothèque d'Apollodore, Besançon/Paris, Les Belles Lettres, 1991, ad III 120, 1.

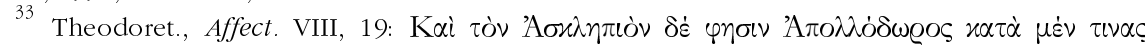

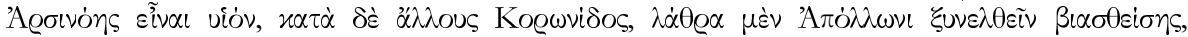

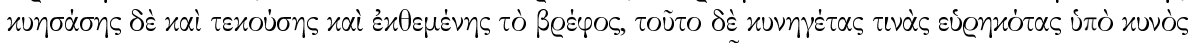

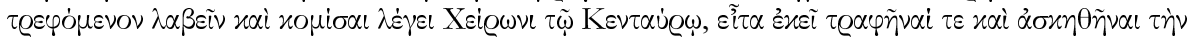

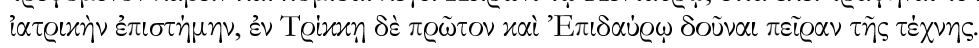


tempi di Omero non aveva ottenuto ancora la deificazione, tanto che il poeta greco faceva guarire le ferite di Ares a Peone ${ }^{34}$ ?

E « chironica », curiosamente, viene definita da Teodoreto la tremenda ferita ai piedi dello Stilita Simeone ${ }^{35}$, che per primo, eroe culturale di un sistema simbolico radicalmente nuovo quale il culto dei santi ${ }^{36}$, inventò il bios sulla colonna, lo stilitismo appunto.

L'aggettivo $\chi \varepsilon \iota \varrho \dot{\omega} \vee \varepsilon \iota 0 \varsigma$, che nella tradizione testimoniata dai paremiografi va ad indicare una ferita ulcerosa ai piedi $^{37}$, si richiama alla ferita purulenta che Eracle causò a Chirone, colpendolo involontariamente con la sua freccia: l'eroe greco - racconta Apollodoro ${ }^{38}$ - tentò invano di curarla, applicando un farmaco fornitogli dallo stesso Chirone; quest'ultimo, immortale ma desiderando porre termine alla propria vita, potrà morire solo quando Prometeo offrirà a Zeus di diventare immortale al suo posto.

Teodoreto del resto utilizza l'aggettivo $\chi \varepsilon \iota \varrho \dot{\omega} \nu \varepsilon \iota 0 \varsigma$, come attributo della ferita di Simeone, in un episodio particolarmente importante della biografia dello stilita quando un diacono, venuto a conoscere gli eccezionali ritmi ascetici del santo, si reca da Simeone e polemicamente gli chiede: $\alpha \dot{\alpha} \nu \varrho \omega \pi \circ \subseteq$ ع $\tilde{\imath}$

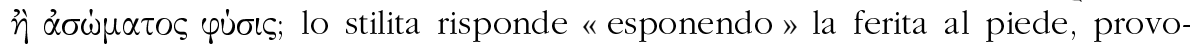
cata dalla stasis sulla colonna:

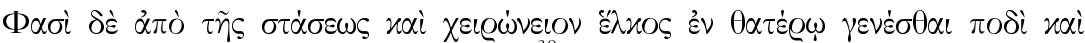

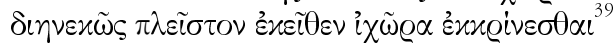

L'aggettivo $\chi \varepsilon \iota @ \dot{\omega} \vee \varepsilon \iota 0 \varsigma^{40}$, oltre a chiarire la localizzazione corporea della ferita - sui piedi -, rafforza quindi la valenza del termine $\varepsilon \lambda \varkappa \varkappa_{0}$ ad indicare la ferita suppurante $^{41}$, da cui continua a fuoriuscire sangue ${ }^{42}$.

34 Theodoret., Affect. VIII 23: "O

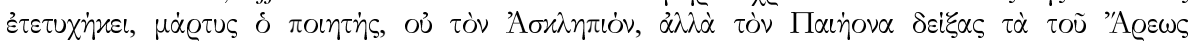

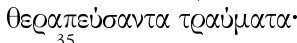

35 A Simeone il Primo Stilita Teodoreto dedica il XXVI capitolo dell'Historia Religiosa.

36 È senz'altro a Peter Brown (in particolare The Cult of the Saints, Chicago-London, Chicago University Press, 1981) che si deve il merito di aver individuato nel culto dei santi un fenomeno essenzialmente nuovo nel panorama della cultura tardo-antica: rifiutando da un lato la tesi della funzione sostitutiva nei confronti dei culti pagani e dall'altro l'idea che il culto dei santi fosse esclusivo appannaggio del popolo, egli ha identificato nei grandi vescovi del V e VI secolo i principali promotori del culto dei santi, e ha evidenziato il modo in cui attorno all'uomo di Dio, ma soprattutto attorno all'uomo di Dio morto, e quindi alla sua tomba e alle sue reliquie, si siano create nuove forme di potere. Proprio sulla figura di Simeone ha calibrato il paradigma dell' holy man nel celeberrimo "The Rise and Function of the Holy Man in Late Antiquity", JRS 61 (1971), p. $80-101$

${ }^{37}$ Così la definisce il paremiografo Zenobio nel II secolo (Zen. VI, 46): X

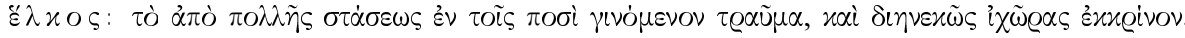

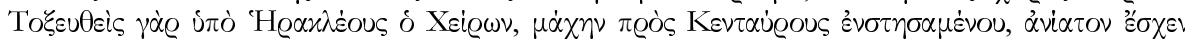

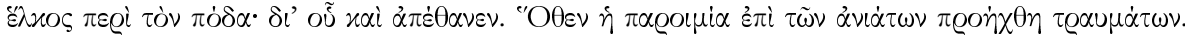

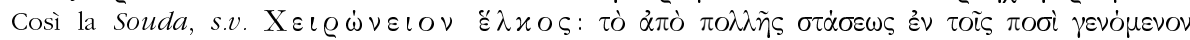

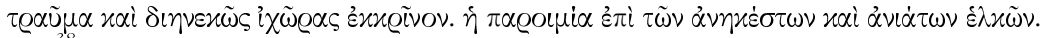

Apollod., Bibl. II, 85

39 Theodoret., Hist. Rel. XXVI, 23, 1-3 
Di fronte al diacono, che gli chiedeva se fosse un uomo o una natura incorporea perché aveva sentito dire che non mangiava e non dormiva, Simeone risponde quindi ordinando di accostare la scala alla sua colonna e di esaminare anzitutto le mani, di passare la mano all'interno dei suoi vestiti e di guardare non solo i piedi ma la sua ferita orribile: l'infermità diviene in questo caso «strumento di realtà », non causa di morte ma modo dell'esistere.

La ferita, la malattia, il dolore, che segnano per gli eroi pagani il passo della fine, divengono per i martiri e per gli asceti il segno della comunione con Dio, di un'imitatio Christi che permette addirittura di ripetere, come nella scena appena citata, l'invito ad esaminare i segni della Passione rivolto dal Salvatore all'incredulo Tommaso.

Può sembrare improprio accostare due opere quali la Graecarum Affectionum Curatio e l'Historia Religiosa, che appartengono a fasi distinte della produzione di Teodoreto e rispondono a scopi differenti; guardare però «sinotticamente» alle rappresentazioni di eroi e santi presenti in esse evidenzia il modo in cui malattie e mutilazioni, febbri e cancrene vengano non solo ad assumere un ruolo «positivo » nel processo di definizione della realtà, ma costituiscano lo stesso campo semantico all'interno del quale Teodoreto mette in scena la battaglia tra vera fede e miti falsi e bugiardi, una battaglia in cui i corpi, egualmente deficitarii o egualmente eccedenti, divengono i «segni » di costellazioni simboliche antitetiche. Così se quelli pagani sono inevitabilmente destinati al fallimento, quelli cristiani «eroicamente » resistono, tanto da poter essere smembrati, come quelli dei martiri, senza perdere unità.

Se Teodoreto nel terzo capitolo della Graecarum Affectionum Curatio afferma che il corpo cristiano è integro e perfetto, nell'VIII ricorda infatti che il corpo cristiano per eccellenza, quello del martire, può pure essere smembrato senza perdere la sua potenza poiché la grazia rimane intera: pertanto anche la più piccola reliquia può contenere la stessa potenza che possederebbe il

40 P. CAnivet, A. Leroy-Molinghen (a cura di), Théodoret de Cyr: histoire des moines de Syrie II, Paris, Les Éditions du Cerf, 1979 (Sources Chrétiennes), p. 207: Canivet traduce " de Chiron ».

${ }^{41}$ È utilizzato in questo senso da Tucidide (II, 49) nel descrivere la sintomatologia della

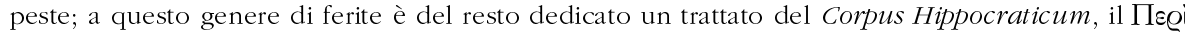
$\hat{\varepsilon} \lambda \varkappa \tilde{\omega} \nu$. Le descrizioni delle malattie e delle ferite all'epidermide ricorrono frequentemente nelle fonti preippocratiche e ippocratiche, cf. J.N. CORVISIER, Santé et société en Grèce ancienne, Paris, Economica, 1985, p. 75: "Ainsi le tableau pathologique présenté par les sources préhippocratiques est-il bien mince: fièvres et malaria, maladies de peau, lèpre blanche, ulcères, cécité. » $C f$. anche p. 106-107, dove l'autore ripercorre i casi di cancrena ai piedi: « Les cas de gangrène sont nombreux dans le corpus hippocratique... On peut aussi distinguer: la nécrose des chairs et peut-être aussi des os, dans le cas d'un talon heurté trop fortement sur le sol, après course sur un chemin raboteux... Un cas de gangrène est cité à la suite d'une douleur au pied. Aucune plaie n'est citée. On a pensé à une gangrène faisant suite à un diabète. L'artérite est aussi possible... Une gangrène est citée à la suite d'incision au pied. Il peut y avoir eu infection... »

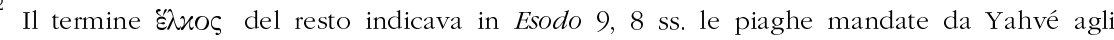
Egiziani; cf. anche Vangelo di Luca 16, 21; Apocalisse 16, 2. 
corpo del martire se non fosse mai stato smembrato. La grazia ripartisce i suoi doni, considerando come unica misura la fede di chi prega ${ }^{43}$.

Se i pagani si scandalizzano di fronte a città e villaggi che si spartiscono i corpi dei martiri ${ }^{44}$, inneggiando loro quali salvatori delle anime e medici dei

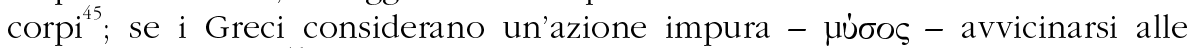
tombe dei martiri ${ }^{46}$ e inorridiscono di fronte al fatto che i santi vengano venerati come protettori, difensori e custodi delle città, essi si trovano ad offrire libagioni a uomini morti e deificati, ad adorare eroi dai corpi deboli e malati, che, incapaci di resistere al dolore, non potranno mai lenire il dolore altrui, tanto deboli da gettarsi nel fuoco per porre fine alla sofferenza invece di resistere alla sofferenza del fuoco, testimoni consunti di un'epoca di cui Teodoreto celebra il tramonto.

Il piano del confronto tra i destinatari del culto - eroi da una parte, santi dall'altra - è ancora quello della corporeità; l'azione di martiri ed asceti è iscritta infatti in quella degli apostoli che con i loro corpi convertirono $i$ popoli, non solo i Romani e i loro sudditi ma anche Persiani, Sciti, Sarmati, Indiani, Etiopi, le genti situate ai confini del mondo ${ }^{47}$. I loro scritti sono nudi di fronte alla raffinatezza retorica ellenica ${ }^{48}$; essi non hanno narrato gesta di eroi, come sofisti esperti ed artificiosi, perché non hanno raccontato di porpora e corone splendenti, di eserciti e di guerre ma di una mangiatoia e di un bambino avvolto in poveri stracci, di un piccolo paese sconosciuto, di povertà, fame, sete, della fatica dei viaggi ma soprattutto della Passione del Cristo, di colpi sul volto e frustate sulle spalle, della colonna e della croce, della morte, quella morte assunta e sopportata fino in fondo dal figlio di Dio, divenuto uomo per la salvezza degli uomini.

E le vicende eroiche degli apostoli prima, come quelle dei martiri e dei santi poi, riattualizzando la sofferenza del Cristo, si fanno per Teodoreto espressione di salvezza: essi hanno offerto la propria schiena a chi voleva fustigarli, il fianco alle torture del fuoco e del ferro, le teste alle spade; si sono lasciati bastonare a morte senza opporre resistenza o mettere al palo, si sono

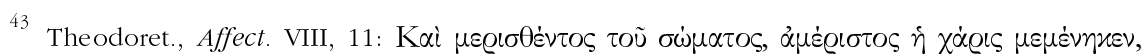

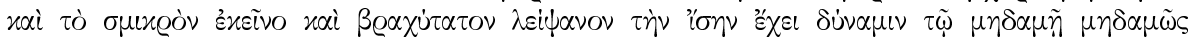

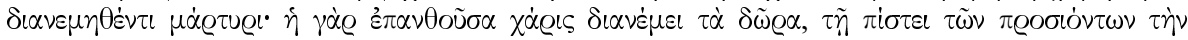

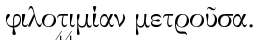

Cf. a tale proposito P. Brown, Il culto dei santi, trad. it. di L. Repici Cambiano, Torino, Einaudi, 2002, p. 15 e ss., dove l'autore ricorda in particolare l' " orrore religioso » dell'imperatore Giuliano di fronte al proliferare dei martiria.

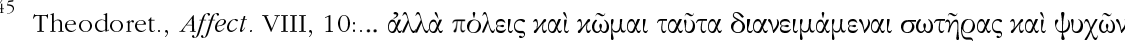

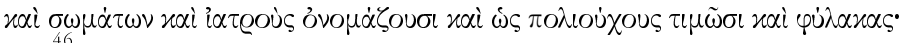

46 Brown, o.c., p. 12: «L'origine del culto cristiano dei santi ebbe luogo nei grandi cimiteri, oltre le mura delle città del mondo romano e per quanto riguarda il contatto con corpi morti, tale culto comportò ben presto il disseppellimento, la traslazione, lo smembrare - per non parlare di chi avidamente toccava e baciava - le ossa dei morti, con la conseguenza che spesso esse furono collocate in aree da cui prima i morti erano esclusi. »

47 Theodoret., Affect. VIII, 6

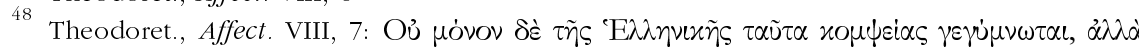

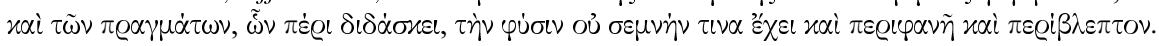


fatti bruciare o hanno assistito con coraggio e fermezza al pasto offerto alle bestie feroci dai loro corpi.

Contrapponendo quei corpi ai corpi eroici celebrati dalla mitologia e dalla retorica ellenica, Teodoreto si fa testimone di un sistema simbolico quale quello della santità, che scardinava e ricomponeva le categorie di comunicazione e di separazione tra umanità e divinità, laddove erano state evidentemente stravolte e rifondate le categorie di separazione e comunicazione tra vita e morte. I cadaveri, che la cultura pagana non poteva avvicinare perché impuri e contaminanti, divengono, nel caso dei martiri e dei santi, pezzi da traslare, da disseppellire, da smembrare ma non solo: ossa e sangue acquistano valore, addirittura valore di scambio; conquistati e sepolti nuovamente, toccati e baciati, i corpi morti dei santi rifondano l'oikoumene, attraverso l'occupazione di luoghi da cui i morti erano precedentemente esclusi e rendendo con la loro stessa presenza «abitabili » spazi primi preclusi all'uomo, deserti o "disumani ${ }^{49}$ »; facendo dimenticare i rimedi illusori di ossa di eroi smarritisi di fronte al dolore, dissoltisi per sempre di fronte al fuoco.

Chiara CREMONESI

Dipartimento di Scienze dell'Antichità

Piazza Capitaniato, 7

I - 35139 PADOVA

\footnotetext{
${ }^{49}$ Brown, o.c., p. 13: Lo stesso confine che « sorgeva fra la città dei vivi e dei morti, finì per essere infranto dall'ingresso delle reliquie e delle loro custodie entro le mura di molte città tardoantiche e dall'ammassarsi di tombe « comuni » attorno ad esse ».
} 